Bull. Austral. Math. Soc.

VOL. 37 (1988) [345-351]

\title{
A LINEAR COMPLEMENTARITY PROBLEM INVOLVING A SUBGRADIENT
}

\author{
J. Parida, A. Sen and A. Kumar
}

\begin{abstract}
A linear complementarity problem, involving a given square matrix and vector, is generalised by including an element of the subdifferential of a convex function. The existence of a solution to this nonlinear complementarity problem is shown, under various conditions on the matrix. An application to convex nonlinear nondifferentiable programs is presented.
\end{abstract}

\section{INTRODUCTION}

For given $M \in \mathbf{R}^{n \times n}$ and $r \in \mathbf{R}^{n}$, the problem of finding an $x \in \mathrm{R}^{n}$ such that

$$
x \geqslant 0, \quad M x+r \geqslant 0, \quad\langle x, M x+r\rangle=0
$$

is called the linear complementarity problem. The existence of solutions for (1.1) has been investigated by many authors (see the references in $[\mathbf{1}]$ ).

We consider the following extension of (1.1). Given a lower semicontinuous positively homogeneous finite convex function $h: \mathbf{R}^{n} \rightarrow \mathbf{R}$, find $x \in \mathbf{R}^{n}$ and $y \in \partial h(x)$ such that

$$
x \geqslant 0, \quad M x+y+r \geqslant 0, \quad\langle x, M x+y+r\rangle=0 .
$$

It may be observed that the problem of finding a stationary point of Kuhn-Tucker type of a nondifferentiable programming problem in which the objective function is the sum of a support function and a quadratic function, and the constraints are linear, becomes a linear complementarity problem of the form (1.2).

A lower semicontinuous positively homogeneous finite convex function is the support function of a certain closed convex set. In particular (Corollary 13.2.1 of [9]) such an $h$ is representable as

$$
h(x)=\max \left\{x^{T} v \mid v \in C\right\}
$$

where

$$
C=\left\{v \in \mathbf{R}^{n}: v^{T} x \leqslant h(x) \text { for all } x\right\} .
$$

Received 20 July, 1987

Copyright Clearance Centre, Inc. Serial-fee code: $0004-9729 / 88 \quad \$ A 2.00+0.00$. 
Note that since $h(x)$ is finite for every $x \in C, C$ is compact (Theorems 8.4 and 13.2 .2 of [0]). Moreover (Corollary 23.5.3 of $[\boldsymbol{\theta}]$ ), one has the subdifferential formula

$$
\partial h(x)=\left\{y \in C: x^{T} y=h(x)\right\}
$$

The representation of a lower semicontinuous positively homogeneous finite convex function as a support function is illustrated $[10]$ in the following two cases:

(i) Let $B$ be a symmetric positive semidefinite matrix. Then $\left(x^{T} B x\right)^{1 / 2}=$ $h(x)$, where $C=\left\{B w: w^{T} B w \leqslant 1\right\}$.

(ii) Let $p$ and $q$ be conjugate exponents; that is, $p^{-1}+q^{-1}=1,1<p<\infty$ and $1<q<\infty$. Let $E$ be a $k \times n$ matrix and let $\|z\|_{p}=\left(\sum_{i=1}^{k}\left|z_{i}\right|^{p}\right)^{1 / p}$. Then $\|E x\|_{p}=h(x)$, where $C=\left\{E^{T} z:\|z\|_{q} \leqslant 1\right\}$.

We give some generalised sets of conditions involving $M$, under each of which there exists a solution to (1.2). Several known classes of matrices $M$ which are relevant to linear complementarity problem (1.1) are seen to satisfy these conditions.

\section{The MaIN Results}

In what follows, we denote by $d$ and $e$ any $n$-vector with all components positive and the $n$-vector with all components unity, respectively.

The following lemma on the variational inequality, which is a special case of Lemma 2.1 of $[8]$, will be the basic tool for establishing our main results.

LEMMA 1. Let $S \subseteq \mathbb{R}^{n}$ be a compact convex set, $r \in \mathbf{R}^{n}, M \in \mathbf{R}^{n \times n}$, and let $h$ be as defined by (1.3). Then there exists an $\bar{x} \in S$, and a $\bar{y} \in \partial h(\bar{x})$ such that

$$
\langle x-\bar{x}, M \bar{x}+\bar{y}+r\rangle \geqslant 0 \text { for all } x \in S \text {. }
$$

THEOREM 1. If the system

$$
\begin{array}{rl}
M_{i} u+t d_{i}=0 & \text { if } u_{i}>0 \\
M_{i} u+t d_{i} \geqslant 0 & \text { if } u_{i}=0 \\
t=-u^{T} M u \geqslant 0 & 0 \neq u \geqslant 0
\end{array}
$$

is inconsistent, then (1.2) has a solution.

Proof: Consider the compact convex sets

$$
S_{\alpha}=\left\{x \in \mathbf{R}^{n}: x \geqslant 0, \quad d^{T} x \leqslant \alpha\right\}
$$

for real $0<\alpha<\infty$. By Lemma 1 , there exists an $x^{\alpha}$ and $y^{\alpha} \in \partial h\left(x^{\alpha}\right)$ such that

$$
\left\langle x-x^{\alpha}, M x^{\alpha}+y^{\alpha}+r\right\rangle \geqslant 0 \quad \text { for all } x \in S_{\alpha},
$$


and applying the duality theory of linear programming, we get a scalar $\xi^{\alpha}$ such that

$$
\begin{gathered}
x^{\alpha} \geqslant 0, \quad M x^{\alpha}+y^{\alpha}+r+\xi^{\alpha} d \geqslant 0 \\
\left\langle x^{\alpha}, M x^{\alpha}+y^{\alpha}+r+\xi^{\alpha} d\right\rangle=0 \\
\xi^{\alpha} \geqslant 0, \quad d^{T} x^{\alpha} \leqslant \alpha, \quad\left(\alpha-d^{T} x^{\alpha}\right) \xi^{\alpha}=0 .
\end{gathered}
$$

We distinguish two cases.

Case 1. $\xi^{\alpha}=0$ for some $\alpha=\bar{\alpha}, 0<\bar{\alpha}<\infty$. It follows from (2.2) and (2.3) that (1.2) has a solution $\left(x^{\bar{\alpha}}, y^{\bar{\alpha}}\right)$.

Case 2. $\xi^{\alpha}>0$ for every $0<\alpha<\infty$. By (2.4), we have $d^{T} x^{\alpha}=\alpha$ for all these $\alpha$. Let $u^{\alpha}=x^{\alpha} / \alpha$. Then $u^{\alpha} \geqslant 0$ and $d^{T} u^{\alpha}=1$. This shows that the set of points $\left(u^{\alpha}, y^{\alpha}\right)$ lies in the compact set $\left\{x: x \geqslant 0, d^{T} x=1\right\} \times C$, and hence, there is a convergent sequence of $\left(u^{\alpha}, y^{\alpha}\right)$ with $\alpha \rightarrow \infty$. Let this sequence be one with $\alpha=\alpha_{1}, \alpha_{2}, \alpha_{3}, \ldots$, or, briefly, with $\alpha \in \Gamma$, and let $(u, y)$ be the limit of the sequence. Clearly, $u \geqslant 0$ and $d^{T} u=1$, which implies $u \neq 0$. Further, from (2.3) and (2.2) respectively, we have

$$
\begin{gathered}
0>-\alpha^{-1} \xi^{\alpha}=\left\langle u^{\alpha}, M u^{\alpha}\right\rangle+\alpha^{-1}\left\langle u^{\alpha}, y^{\alpha}+r\right\rangle, \\
M u^{\alpha}+\alpha^{-1}\left(y^{\alpha}+r\right)+\left(\alpha^{-1} \xi^{\alpha}\right) d \geqslant 0
\end{gathered}
$$

for all $\alpha \in \Gamma$, which in the limit gives $0 \geqslant u^{T} M u=-t$ (say) and $M u+t d \geqslant 0$. Since $d^{T} u=1$, we also have $\langle u, M u+t d\rangle=0$. This shows that $u$ is a solution to the system (2.1), contradicting the assumption of the theorem. Hence, $\xi^{\alpha}=0$ for at least one $\alpha$.

The following corollary is a consequence of Theorem 1 and the definitions of the matrices involved. For the definitions, we refer to Eaves [2] and Karamardian [3].

COROLLARY 1. There exists a solution to (1.2) for every $r \in \mathbf{R}^{n}$ if $M$ is any of the following matrices: positive definite, strictly copositive, $P$-matrix, strictly semimonotone and regular matrix (for a regular matrix, take $d=e$ in (2.1)).

THEOREM 2. If there is a $\bar{u} \geqslant 0$, and a scalar $\beta>d^{T} \bar{u}$ such that

$$
\min \{\langle x-\bar{u}, M x+y+r\rangle \mid y \in \partial h(x)\} \geqslant 0
$$

for every $x \in\left\{x: x \geqslant 0, \quad d^{T} x=\beta\right\}$, then (1.2) has a solution.

Proof: Consider the set $S_{\beta}=\left\{x: x \geqslant 0, d^{T} x \leqslant \beta\right\}$. Clearly, $S_{\beta}$ is compact and convex. Now, applying Lemma 1 , and then proceeding as in the proof of Theorem 
1, we get vectors $\bar{x} \in \mathbf{R}^{n}, \bar{y} \in \partial h(\bar{x})$, and a scalar $\bar{\xi}$ such that

$$
\begin{gathered}
\bar{x} \geqslant 0, \quad M \bar{x}+\bar{y}+r+\bar{\xi} d \geqslant 0 \\
\langle\bar{x}, M \bar{x}+\bar{y}+r+\bar{\xi} d\rangle=0 \\
\bar{\xi} \geqslant 0, \quad d^{T} \bar{x} \leqslant \beta, \quad\left(\beta-d^{T} \bar{x}\right) \bar{\xi}=0 .
\end{gathered}
$$

If $\bar{\xi}=0$, then $(\bar{x}, \bar{y})$ solves (1.2). Assume that $\bar{\xi}>0$, and by (2.8), we have $d^{T} \bar{x}=\beta$. Consequently, from (2.5)-(2.7), it follows that

$$
0 \leqslant\langle\bar{x}-\bar{u}, M \bar{x}+\bar{y}+r\rangle \leqslant\left(d^{T} \bar{u}-\beta\right) \bar{\xi}<0,
$$

a contradiction. Therefore, we conclude that $\bar{\xi}=0$.

As a corollary of Theorem 2, we get the following result for a positive semidefinite matrix $M$, which can also be obtained by specialising to the present case the result of McLinden [4] for monotone multifunctions in a general setting.

Corollary 2. If $M$ is positive semidefinite and there exists a $\bar{u} \geqslant 0$, and a $\bar{v} \in \partial h(\bar{u})$ such that $M \bar{u}+\bar{v}+r>0$, then (1.2) has a solution.

PRoof: Set $d=M \bar{u}+\bar{v}+r$, and then choose a scalar $\beta>d^{T} \bar{u}$. Now, for any $x \geqslant 0$ wiht $d^{T} x=\beta$, it follows from the positive semidefiniteness of $M$ and the definition of a subgradient that

$$
\begin{aligned}
\langle x-\bar{u}, M x+y+r\rangle & \geqslant\langle x-\bar{u}, M \bar{u}+\bar{v}+r\rangle \\
& =d^{T}(x-\bar{u})=\beta-d^{T} \bar{u}>0
\end{aligned}
$$

for all $y \in \partial h(x)$. Thus, the conditions of Theorem 2 are satisfied.

The next corollary gives an existence result for the class of copositive matrices, which includes as a subclass the class of copositive plus matrices [2, p. 621].

COROLlaRY 3. If $M$ is a copositive matrix and $r^{T} x+h(x) \geqslant 0$ for every $x \geqslant 0$ with $e^{T} x=1$, then (1.2) has a solution.

Proof: The result follows immediately from Theorem 2 by setting $\bar{u}=0, \beta=1$ and $d=e$.

\section{AN APPLICATION}

In a number of mathematical programming problems studied in detail, such as those in $[5,6,7,10]$, the objective function is the sum of a lower semicontinuous positively homogeneous finite convex function and a differentiable convex function, while the constraint functions are differentiable. Below we consider a special case in which the 
objective function is the sum of a support function and a quadratic function. Though the objective function is not differentiable, the simple form of the subdifferential of a support function is helpful in framing a stationary point problem of Kuhn-Tucker type for this problem.

Let $D \in \mathbf{R}^{n \times n}$ be a symmetric positive semidefinite matrix, $A \in \mathbf{R}^{m \times n}, c \in \mathbf{R}^{n}$, $b \in \mathbf{R}^{m}$ and let $h$ be defined by (1.3). The problem then is as follows:

$$
\begin{array}{r}
(\mathrm{P}): \text { Minimise } Q(x)=\frac{1}{2} x^{T} D x+c^{T} x+h(x) \\
\text { subject to } A x-b \geqslant 0, \quad x \geqslant 0 .
\end{array}
$$

It can easily be checked that if there exists $x \in \mathbf{R}^{\mathbf{n}}, \lambda \in \mathbf{R}^{\mathbf{m}}$ and $y \in \partial h(x)$, satisfying

$$
\begin{gathered}
x \geqslant 0, \quad \lambda \geqslant 0 \\
A x-b \geqslant 0, \quad D x-A^{T} \lambda+y+c \geqslant 0 \\
\langle\lambda, A x-b\rangle=0, \quad\left\langle x, D x-A^{T} \lambda+y+c\right\rangle=0
\end{gathered}
$$

then $x$ is an optimal solution of $(\mathrm{P})$. Now we define

$$
\begin{aligned}
& M=\left[\begin{array}{cc}
D & -A^{T} \\
A & 0
\end{array}\right] \quad r=\left[\begin{array}{c}
c \\
-b
\end{array}\right] \\
& h_{0}(x, \lambda)=h(x)+0
\end{aligned}
$$

for each $(x, \lambda) \in \mathbb{R}^{n+m}$, and note that $(y, s) \in \partial h_{0}(x, \lambda)$ if and only if $y \in \partial h(x)$ and $s=0$. Taking $M, r$ and $h_{0}(x, \lambda)$ as above, the stationary point problem (3.1) can be projected into a complementarity problem of the form (1.2). Clearly, $M$ in (3.2) is positive semidefinite. An application of Corollary 2 yields the following theorem.

Theorem 3. If there exist $\hat{x} \in \mathbf{R}^{n}, \hat{\lambda} \in \mathbf{R}^{m}$ and $\hat{y} \in \partial h(\hat{x})$ such that

$$
\begin{gathered}
\hat{x} \geqslant 0, \quad \hat{\lambda} \geqslant 0 \\
A \hat{x}-b>0, \quad D \hat{x}-A^{T} \hat{\lambda}+\hat{y}+c>0
\end{gathered}
$$

then $(P)$ has an optimal solution.

\section{Numerical EXAMPLES}

We give below some examples to illustrate the existence results of Sections 2 and 3.

Example 1. Let $h(x)=\left(x^{T} B x\right)^{1 / 2}$,

$$
M=\left[\begin{array}{ll}
-1 & 1 \\
-2 & 1
\end{array}\right], \quad r=\left[\begin{array}{c}
-\sqrt{3} / 2 \\
1-\sqrt{3}
\end{array}\right], \quad B=\left[\begin{array}{ll}
1 & 0 \\
0 & 1
\end{array}\right] .
$$

Here $M$ is a regular matrix (see $[3$, p. 126]). By Corollary $1,(1.2)$ has a solution, and we see that $x=(1 / 2, \sqrt{3} / 2), y=(1 / 2, \sqrt{3} / 2)$ is a solution. 
Example 2. Let $h(x)$ be as in Example 1, and let

$$
M=\left[\begin{array}{cc}
1 & -1 \\
-1 & 1
\end{array}\right], \quad r=\left[\begin{array}{c}
-2 \\
3
\end{array}\right]
$$

Here $M$ is positive semidefinite, and, for $\bar{u}=(2,0), \bar{v}=(1,0) \in \partial h(\bar{u})$, we have $M \bar{u}+\bar{v}+r>0$. By Corollary 2, (1.2) has a solution, and we see that $x=(1,0)$, $y=(1,0)$ is a solution.

Example 3. Let $h(x)=\|E x\|_{2}$,

$$
M=\left[\begin{array}{ll}
0 & 1 \\
1 & 0
\end{array}\right], \quad r=\left[\begin{array}{c}
-\sqrt{2} \\
1
\end{array}\right], \quad E=\left[\begin{array}{cc}
1 & 1 \\
1 & -1
\end{array}\right] .
$$

Here $M$ is copositive, and, for $x \geqslant 0$ with $e^{T} x=1, r^{T} x+h(x)$ has values between zero and $(1+\sqrt{2})$. Consequently, Corollary 3 ensures the existence of a solution, and we find that $x=(1,0), y=(\sqrt{2}, 0)$ is a solution of $(1.2)$.

Example 4. Let $h(x)$ be as in Example 1. Consider the problem: minimise $Q(x)=$ $-x_{1}-x_{2}+h(x)$ over $x_{1}>0,0 \leqslant x_{2} \leqslant 1$. It can easily be seen that -1 is the infimum of $Q(x)$ over the constraint set, but the problem has no optimal solution. Consequently Theorem 3 implies that the system (3.3) cannot be consistent. In fact, we need $\hat{\lambda} \geqslant 0,\left(\hat{y}_{1}, \hat{y}_{2}\right) \in \partial h(\hat{x})$ such that $\hat{y}_{1}>1$ and $\hat{\lambda}+\hat{y}_{2}>1$, which is not possible, since $\hat{y}_{1}^{2}+\hat{y}_{2}^{2} \leqslant 1$.

\section{REFERENCES}

[1] A. Berman, 'Matrices and the linear complementarity problem', Linear Algebra Appl. 40 (1981), 249-256.

[2] B.C. Eaves, 'The linear complementartiy problem', Management Sci. 17 (1971), 612-634.

[3] S. Karamardian, 'The complementarity problem', Math. Programming 2 (1972), 107-129.

[4] L. Mclinden, 'The complementarity problem for maximal monotone multifunctions', in Variational Inequalities and Complementarity Problems, ed. R.W. Cottle, F. Gianessi and J.L. Lions, pp. 251-270 (John Wiley and Sons, Chichester, 1980).

[5] B. Mond, 'A class of nondifferentiable mathematical programming problems', J. Math. Anal. Appl. 46 (1974), 169-174.

[6] B. Mond and M. Schecter, 'A programming problem with an $L_{p}$ norm in the objective function', J. Austral. Math. Soc. Ser. B 10 (1976), 333-342.

[7] J. Parida and A. Sen, 'Duality and existence theory for nondifferentiable programming', $J . O p$. tim. Theory Appl. 48 (1986), 451-458.

[8] J. Parida and A. Sen, 'A class of nonlinear complementarity problem for multifunctions', J. Optim. Thoery Appl. 53 (1987), 105-113.

[9] R.T. Rockafellar, Convex Analysis (Princeton University Press, Princeton, New Jersey, 1969).

[10] M. Schechter, 'A subgradient duality theorem', J. Math. Anal. Appl. 61 (1977), 850-855. 
Dr. J. Parida,

Department of Mathematics, Regional Engg. College,

Rourkela 769008

India

Dr. A. Kumar

Lecturer in Mathematics

Vedvyas College

Rourkela - 769041

India
Dr. A. Sen

Lecturer in Mathematics

SKDAV Women's College

Rourkela

India 